\title{
CHARACTERIZATIONS OF ELEMENTARY OPERATORS
}

\author{
Charalampos Magiatis
}

Abstract. Let $\mathscr{A}$ be an ultraprime algebra and $\mathscr{I}$ a closed ideal in $\mathscr{A}$ with left (resp. right) approximate unit. We characterize elementary operators on $\mathscr{A}$ in terms of their images. We show that if $\Phi$ is an elementary operator on $\mathscr{A}$, then the set $\Phi\left(\mathscr{A}_{1}\right)$ (where $\mathscr{A}_{1}$ is the unit ball of $\mathscr{A}$ ) is a left (resp. right) uniformly approximable subset of $\mathscr{I}$ if and only if for any minimal length representation $\sum_{i=1}^{k} M_{a_{i}, b_{i}}$ of $\Phi$ we have $\left\{a_{i}\right\}_{i=1}^{k} \subseteq \mathscr{I}$ (resp. $\left\{b_{i}\right\}_{i=1}^{k} \subseteq \mathscr{I}$ ).

Mathematics subject classification (2020): 47L05.

Keywords and phrases: Elementary operator, ultraprime algebra, prime $C^{*}$-algebra, uniformly approximable set.

\section{REFERENCES}

[1] L. ARAMBAŠIĆ AND I. Gogić, Elementary operators on Hilbert modules over prime $C^{*}$-algebras, J. Math. Anal. Appl. 485, 2 (2020), 10 pp.

[2] M. Bresar AND Y. V. Turovs KiI, Compactness conditions for elementary operators, Studia Math. 178, 1 (2007), 1-18.

[3] T. S. ERickson, W. S. Martindale 3Rd And J. M. Osborn, Prime nonassociative algebras, Pacific J. Math. 60, 1 (1975), 49-63.

[4] C. K. Fong And A. R. Sourour, On the operator identity $\sum A_{k} X B_{k} \equiv 0$, Canad. J. Math. 31, 4 (1979), 845-857.

[5] E. C. LANCE, Hilbert $C^{*}$-Modules: A toolkit for operator algebraists, London Mathematical Society Lecture Note Series 210, Cambridge University Presss, Cambridge, 1995.

[6] C. Magiatis, Elementary operators on the algebra of adjointable operators on a Hilbert module, J. Math. Anal. Appl. 475, 1 (2019), 628-640.

[7] M. MathieU, Elementary operators on prime $C^{*}$-algebras, II, Glasg. Math. J. 30, 3 (1988), 275-284.

[8] M. Mathieu, Elementary operators on prime $C^{*}$-algebras, I, Math. Ann. 284, 2 (1989), 223-244.

[9] M. Mathieu, Rings of quotients of ultraprime Banach algebras, with applications to elementary operators, in: Conference on Automatic Continuity and Banach Algebras (Canberra, 1989), Proc. Centre Math. Anal. Austral. Nat. Univ. 21, Austral. Nat. Univ., Canberra, 1989, 297-317.

[10] G. J. Murphy, $C^{*}$-Algebras and Operator Theory, Academic Press, Boston, 1990.

[11] V. S. Shulman And YU. V. TurovsKiI, Topological radicals and joint spectral radius, Funktsional. Anal. i Prilozhen. 46, 4 (2012), 61-82 (in Russian); English transl.: Funct. Anal. Appl. 46, 4 (2012), 287-304.

[12] R. M. TIMONEY, Some formulae for norms of elementary operators, J. Operator Theory 57, 1 (2007), $121-145$.

[13] K. VAla, On compact sets of compact operator, Ann. Acad. Sci. Fenn. Ser. A I 351 (1964), 1-8.

[14] G. A. WILLIs, Ultraprime group algebras, Proc. Centre Math. Anal. Austral. Nat. Univ. 21 (1989), 345-349. 